\title{
Isquemia intestinal secundaria a una trombosis venosa
}

\author{
Intestinal ischemia secondary to venous thrombosis \\ Carolina Andrea Pérez Cruz, * Francisco Julián Rangel Gámez, * \\ Carolina González Vergara, ${ }^{*}$ Lizbett Hidalgo Pérez ${ }^{\ddagger}$
}

La trombosis venosa es la etiología de menor frecuencia en la isquemia intestinal, la cual tiene una incidencia de $5-15 \%{ }^{1}$ Es la cuarta causa de presentación por lo que se debe conocer el panorama de esta etiología, ya que provoca una mortalidad de más de $60 \%$, si no se realiza un diagnóstico temprano, esto es, un diagnóstico menor a 12 horas. $^{2,3}$

Se ha visto que la presentación de la isquemia intestinal por trombosis venosa tiene una mayor incidencia en pacientes varones entre la quinta y sexta década de la vida; está asociada con factores de riesgo como estados de hipercoagulabilidad (embarazo, neoplasia, síndrome y antifosfolípidos) y otros menos comunes como falla cardiaca derecha, hipertensión portal, condiciones inflamatorias intraabdominales (pancreatitis, diverticulitis), trauma y obesidad. Hasta 37\% suele ser idiopática y cabe mencionar que más de $80 \%$ de los pacientes presentan más de un factor de riesgo. $1,2,4$

La isquemia intestinal es una patología ocasionada por hipoxia en el intestino, secundaria a un descenso abrupto en la perfusión. ${ }^{4}$ Existe una reducción del retorno venoso, el cual producirá edema de la pared intestinal, alteración de la perfusión microvascular y aumento de la presión venosa, lo que llevará posteriormente a un infarto segmentario por afectación del eje porto-esplénico-mesentérico. La formación de trombosis venosa ocurre mayormente en la vena mesentérica superior (70\%) y secundariamente en la vena mesentérica inferior y vena porta (30\%). La isquemia
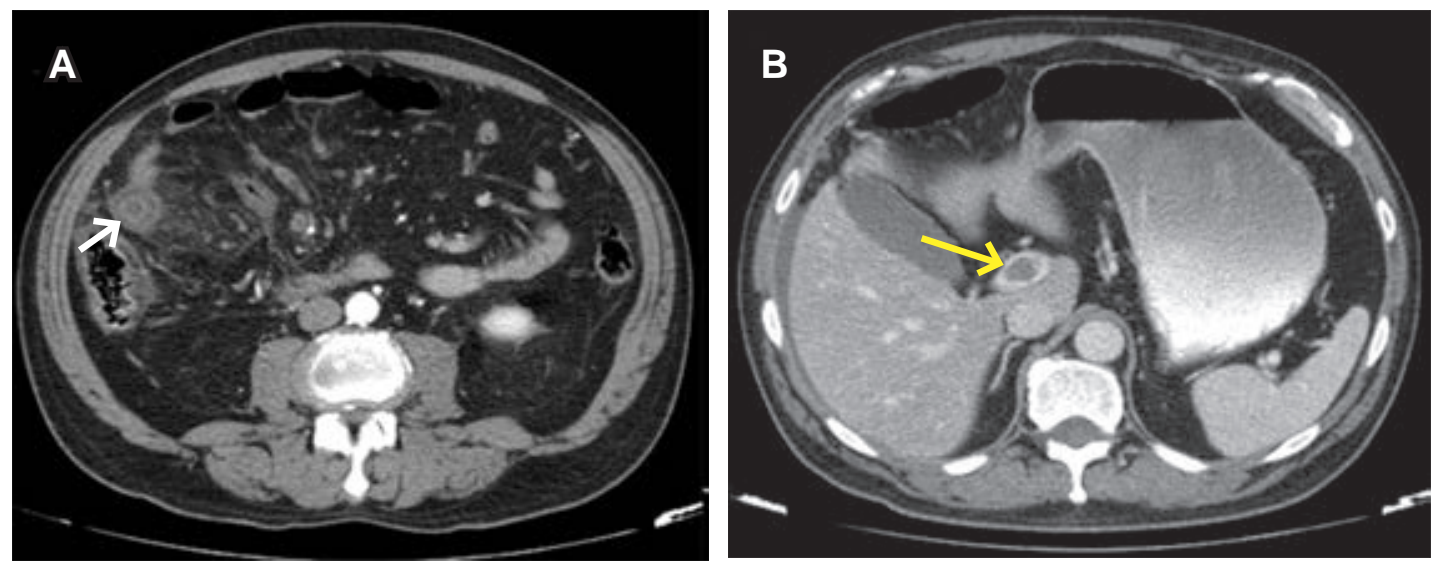

Figura 1: Cortes axiales de tomografía contrastada de abdomen. (A) Fase arterial: se observan datos de isquemia intestinal que consisten en asa con engrosamiento de pared de $6.5 \mathrm{~mm}$ o "signo de diana" (flecha), pobre reforzamiento periférico anular postcontraste y paniculitis a su alrededor. (B) Fase portovenosa: se demuestra trombo intraluminal en el tronco de la vena porta (flecha) y líquido libre perihepático.

* Departamento de Radiología e Imagen.

₹ Departamento de Anatomía Patológica.

Hospital Ángeles Mocel, Ciudad de México.

www.medigraphic.com/actamedica
Correspondencia:

Carolina Andrea Pérez Cruz

Correo electrónico: caroolina_0793@hotmail.com

Aceptado: 26-09-2019.

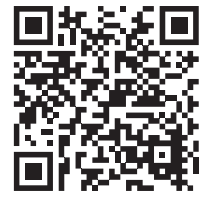


que provoca la trombosis venosa incide en su mayor parte en el íleon (64-83\%), seguido del yeyuno (50-81\%) y en menor proporción en el duodeno (4-8\%). ${ }^{1,3}$

El diagnóstico clínico temprano se considera difícil por la inespecificidad del cuadro, el cual abarca un espectro muy amplio y puede confundirse con múltiples diagnósticos diferenciales. El síntoma más común es el dolor abdominal tipo cólico que se presenta en $100 \%$ de los pacientes; pueden o no estar presentes vómito, diarrea y sangre en las heces $y$, si el cuadro progresa, puede existir inestabilidad hemodinámica más datos de respuesta inflamatoria sistémica.

Los estudios de laboratorio pueden ser tan inespecíficos como el cuadro clínico y se puede esperar leucocitosis, acidosis metabólica y elevación de los marcadores de respuesta inflamatoria. ${ }^{1-4}$
Los estudios de imagen son los indicados para el diagnóstico. El papel de la radiografía simple se limita a demostrar dilatación de asas y presencia de niveles hidroaéreos, aunque el observar neumatosis intestinal y aire libre incrementará el diagnóstico de certeza. Sin embargo, en $25 \%$ de los pacientes puede observarse una radiografía normal. $^{2}$

La utilización de ultrasonido Doppler podría ser una buena opción, pues se ha reportado que tiene una especificidad hasta de $85 \%$; sin embargo, la falla diagnóstica es considerable, al ser operador dependiente. ${ }^{1}$

El estándar de oro en el diagnóstico de isquemia mesentérica de origen trombótico es la angiotomografía, la cual tiene una sensibilidad de $93 \%$ y especificidad de $95 \%$.

El diagnóstico tomográfico se realiza valorando el engrosamiento mayor a $3 \mathrm{~mm}$ de la pared intestinal del asa afec-
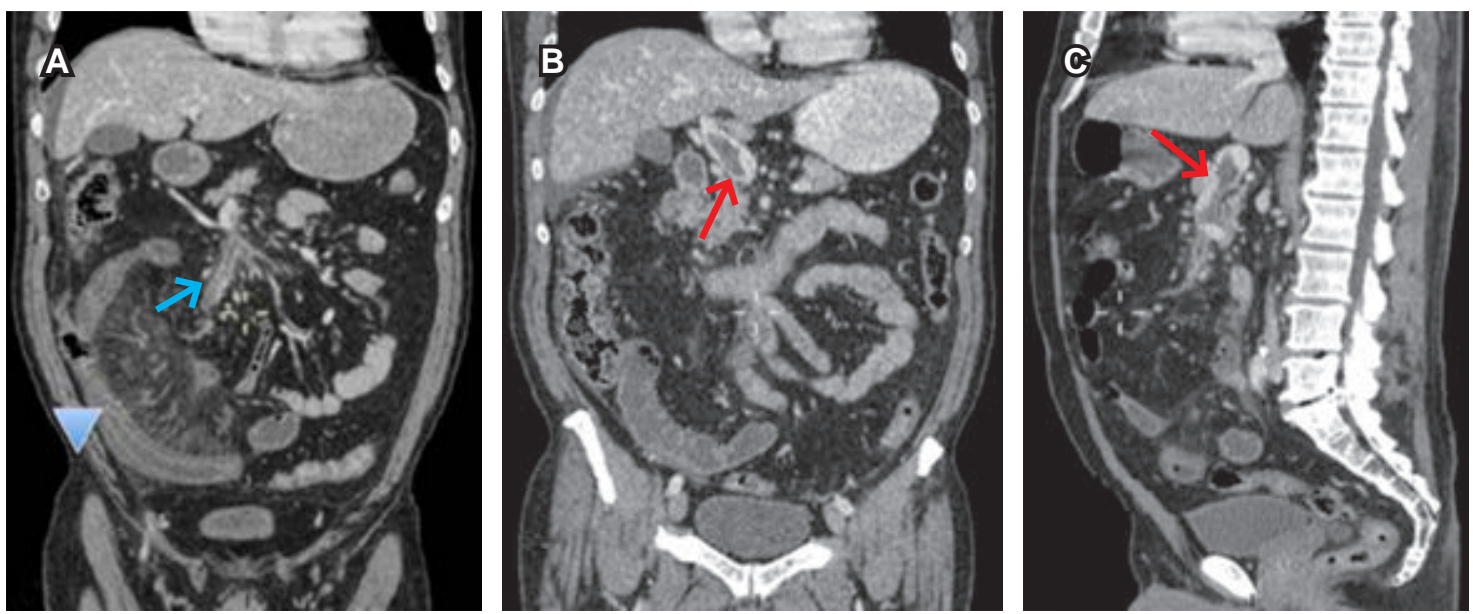

Figura 2: Reconstrucciones de tomografía contrastada de abdomen. (A) Corte coronal en fase arterial que demuestra asa intestinal de yeyuno distal con datos de isquemia, engrosamiento de pared y ausencia de reforzamiento postcontraste (punta de flecha). Se observa trombo oclusivo en la vena mesentérica superior (flecha). (B) Corte coronal y (C) corte sagital en fase venosa que demuestran el trombo en la vena porta (flecha).
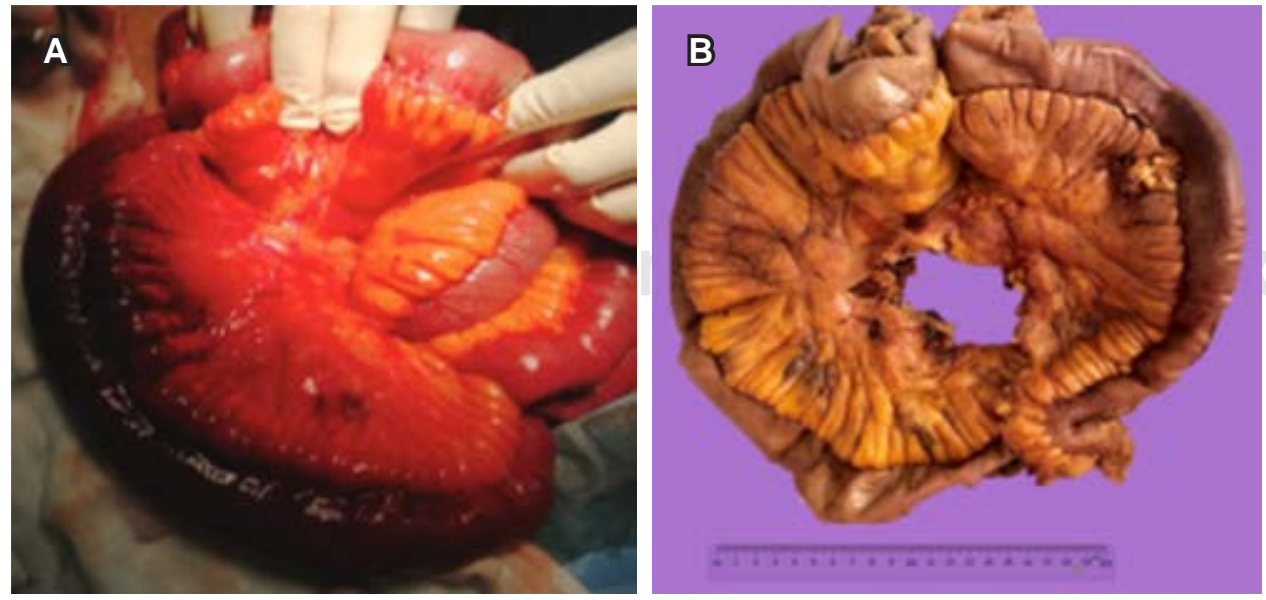

Figura 3:

(A) Transoperatorio con cambio significativo en la coloración y evidencia de yeyuno distal isquémico. (B) Pieza macroscópica del segmento de intestino delgado resecado de 133 $\times 3 \mathrm{~cm}$ con trombosis mesentérica venosa. 

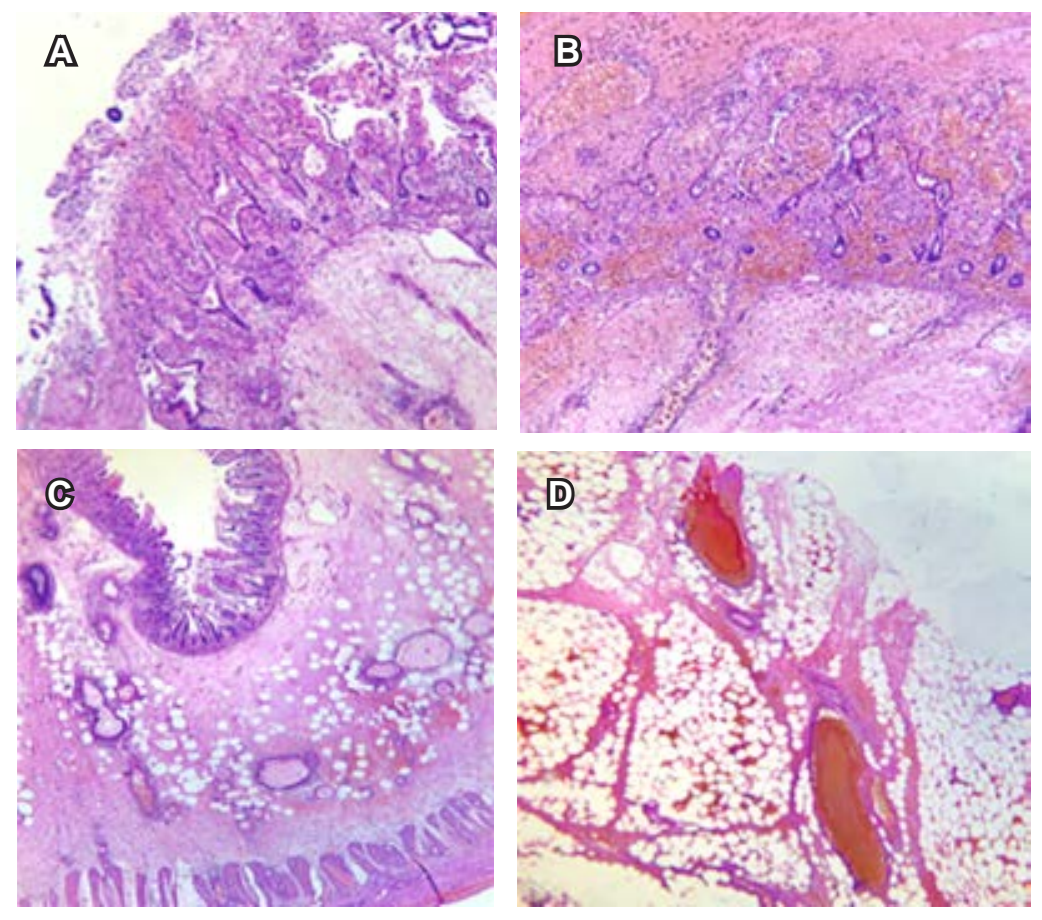

Figura 4:

Tinción hematoxilina y eosina, aumento $4 \mathrm{X}$. (A) Necrosis de la mucosa. (B) Necrosis transmural. (C) Zona de transición entre la necrosis y el tejido viable donde se observa hemorragia isquémica. (D) Hemorragia isquémica en el tejido adiposo secundaria a trombos. tada, el edema submucoso intestinal descrito como "signo de diana", la ausencia de reforzamiento postcontraste, neumatosis intestinal, aire libre, ascitis, congestión venosa y defectos de llenado por la presencia de trombos intraluminales en la fase venosa del estudio contrastado., $1,5,6$

El tratamiento definitivo es la resección quirúrgica temprana más anticoagulación. Los factores de mal pronóstico consideran la edad avanzada, choque séptico, antecedente de infarto agudo al miocardio menor a seis meses, insuficiencia renal y trombosis de la vena porta. ${ }^{6}$

Se presenta el caso de un hombre de 63 años con diagnóstico de isquemia intestinal que es secundaria a trombosis venosa; el paciente es obeso, fumador, diabético tipo 2 e hipertenso de larga evolución. Cursó con dolor abdominal difuso tipo cólico, el cual luego de siete días se agudizó y localizó en el hemiabdomen derecho, con un puntaje en la escala visual análoga (EVA) de 10/10, acompañado de náusea y diaforesis.

En los estudios de laboratorio se documenta acidosis metabólica y leucocitosis de 20,000 por campo.

La alta mortalidad de la isquemia mesentérica venosa, aunque de baja incidencia, requiere de un manejo oportuno, por lo que el diagnóstico juega un papel elemental.

Los estudios de imagen no deben ser postergados en pacientes con factores de riesgo, aun con un cuadro clínico inespecífico. La angiotomografía debe ser el estudio de elección de primera línea.

\section{REFERENCIAS}

1. Trejo Ávila ME, Arce Liévano E, Cuendis Velázquez A, Romero Loera LS. Síndrome abdominal agudo por trombosis venosa mesentérica y portal. Informe de un caso y revisión del tema. Rev Fac Med [Internet]. 2017; 60 (1): 23-27. Disponible en: https://www.medigraphic.com/ cgibin/new/resumen.cgi?IDARTICULO=70504.

2. Motta-Ramírez GA, Sánchez-García JC, Ontiveros-Rodríguez A López-Ramírez MA, Rebollo-Hurtado V et al. Isquemia mesentérica aguda: urgencia que exige un abordaje diagnóstico integral. An Radiol Méx. 2015; 14 (1): 66-88. Disponible en: https://www.medigraphic. com/pdfs/anaradmex/arm-2015/arm151f.pdf

3. Del Río Solá ML, González-Fajardo JA, Vaquero Puerta C. Isquemia mesentérica aguda. Diagnóstico y tratamiento. Angiologia [Internet]. 2015; 67 (2): 133-139. Disponible en: http://dx.doi.org/10.1016/j. angio.2014.05.015.

4. Menke J. Diagnostic accuracy of multidetector CT in acute mesenteric ischemia: systematic review and meta-analysis. Radiology. 2010; 256 (1): 93-101. Disponible en: https://pubs.rsna.org/doi/pdf/10.1148/ radiol.10091938

5. Ravipati M, Katragadda S, Go B, Zarling EJ. Acute mesenteric ischemia: a diagnostic challenge in clinical practice. Pract Gastroenterol. 2011; 35 (8): 35-43. Disponible en: https://pdfs.semanticscholar.org/db0f/ 82cd18197dc2e67388e81e3852e0f86216a7.pdf

6. Guerrero SV. Isquemia mesentérica aguda. Revista Médica Sinergia. 2017; 2 (10): 7-11. Disponible en: https://www.medigraphic.com/ pdfs/sinergia/rms-2017/rms1710b.pdf 